\title{
Paradigm of the time-resolved magneto-optical Kerr effect for femtosecond magnetism
}

\author{
G. P. Zhang ${ }^{1 \star}$, W. Hübner ${ }^{2}$, Georgios Lefkidis ${ }^{2}$, Yihua Bai ${ }^{3}$ and Thomas F. George ${ }^{4}$
}

The magneto-optical Kerr effect (MOKE) is a powerful tool for studying changes in the magnetization of ferromagnetic materials. It works by measuring changes in the polarization of reflected light. However, because the conventional theoretical basis for interpreting a MOKE signal assumes measurement with continuous-wave light ${ }^{1,2}$, its use for understanding highspeed magnetization dynamics of a material probed with femtosecond optical pulses ${ }^{3,4}$ has been controversial ${ }^{5-10}$. Here we establish a new paradigm for interpreting timeresolved MOKE measurements, through a first-principles investigation of ferromagnetic nickel. We show that the timeresolved optical and magnetic responses energetically follow their respective optical and magneto-optical susceptibilities. As a result, the one-to-one correspondence between them sensitively depends on the incident photon energy. In nickel, for photon energies below $2 \mathrm{eV}$ the magnetic response is faithfully reflected in the optical response, but above $2 \mathrm{eV}$ they decouple. By constructing a phase-sensitive polarization versus magnetization plot, we find that for short pulses the magnetic signals are delayed by $10 \mathrm{fs}$ with respect to the optical signals. For longer pulses, the delay shortens and the behaviour approaches the continuous-wave response. This finally resolves the long-standing dispute over the interpretation in the time-resolved MOKE measurements and lays a solid foundation for understanding femtomagnetism ${ }^{3,4}$.

Laser-induced femtosecond magnetism ${ }^{3}$ or femtomagnetism opens a new frontier for a faster magnetic storage device ${ }^{4,11}$, but probing such a fast magnetization change is a big challenge experimentally and theoretically ${ }^{12-14}$. Shortly after its discovery, second-harmonic generation was used to probe the ultrafast spin dynamics and revealed a faster magnetization dynamics of $300 \mathrm{fs}$ (ref. 15), but a similar study showed that the magnetooptical second-harmonic-generation signal change does not reflect magnetization change on a subpicosecond timescale ${ }^{16}$. A very recent X-ray magnetic circular dichroism study showed an even faster $(120 \mathrm{fs})$ demagnetization ${ }^{10}$. The majority of experimental investigations are based on the time-resolved magneto-optical Kerr effect (TRMOKE), inspired by the huge success of the static magneto-optical Kerr effect in probing magnetism; however, whether the ultrafast TRMOKE signal reflects the magnetization change is still under debate. Koopmans et al. ${ }^{5}$ concluded from the difference between the Kerr ellipticity and rotation that the loss of magneto-optical contrast cannot directly be related to an instantaneous demagnetization, and other experiments showed no or only negligible difference between ellipticity and rotation ${ }^{6-9}$. Up to now, although the experimental results start to converge, they leave behind a fundamental question: what does TRMOKE really probe, a simple optical excitation artefact (charge origin) or a genuine magnetic excitation (spin origin)? Failing to answer this question significantly affects our confidence in TRMOKE as a vital tool to probe ultrafast magnetization, particularly femtomagnetism ${ }^{17-19}$. Thus, a deep theoretical understanding is imperative. Our first attempt was made using a model Hamiltonian $^{12,20}$, long before these new experimental results, and the magnetization occurs much faster than those observed experimentally, as our laser pulse is much shorter. The study by Oppeneer and Liebsch removed electrons from the valence band to conduction band to simulate the excitation ${ }^{21}$ and did not compare optical and magnetic responses, and neither did Vernes and Weinberger who developed a linear response theory ${ }^{22}$. Up to now, no existing theories ${ }^{21,22}$, including our latest published studies ${ }^{23-25}$, have addressed this crucial question in the time domain.

A typical TRMOKE experiment measures ellipticity and rotation of the outgoing light beam with respect to the incident beam as a function of time, with a sample experimental geometry shown schematically in Fig. 1. The hope is that a change in ellipticity and rotation, which is an optical signal, links to the true magnetization in a sample. Its theoretical basis is that both the magnetic and optical responses share the common time-dependent density matrix $\rho_{\mathbf{k}}(t)$ through $\langle M(t)\rangle=\sum_{\mathbf{k}} \operatorname{Tr}\left[\rho_{\mathbf{k}}(t) S_{\mathbf{k}}\right]$ and $\langle P(t)\rangle=\sum_{\mathbf{k}} \operatorname{Tr}\left[\rho_{\mathbf{k}}(t) D_{\mathbf{k}}\right]$, respectively, where the summation is over the crystal momentum $\mathbf{k}$, but, as the spin matrix $S$ and dipole matrix $D$ differ, their intrinsic correlation is masked by taking their traces. Here we aim to establish such a correlation by carrying out a massively parallel ${ }^{26}$ and first-principles simulation ${ }^{27}$ in ferromagnetic nickel ${ }^{24,25}$, first solving nearly half a million (k points) Liouville equations of density matrices (see Supplementary Information), and then carefully comparing the polarization with magnetization ${ }^{25}$. Our theory does not include the electron correlation effect ${ }^{12}$ beyond the density functional theory and is valid for a weak laser field in a temporal region before thermalization. We choose a Gaussian-shaped laser pulse with a duration of $12 \mathrm{fs}$ and a laser field amplitude of $0.05 \mathrm{~V} \AA^{-1}$. Figure $2 \mathrm{a}, \mathrm{b}$ presents a first and comprehensive picture of the time-dependent optical and magnetic responses as a function of the incident photon energy. The open-circled line denotes the results obtained with the experimental laser energy ${ }^{3}$ of $2 \mathrm{eV}$, and all the curves are vertically shifted for a better view. The first-order off-diagonal polarization $\left(\operatorname{Im}\left[P_{x y}^{(1)}\right]\right)$, which is responsible for the magneto-optical Kerr effect, shows a systematic change (Fig. 2a). On the short timescale it consistently shows a valley at time $t=0 \mathrm{fs}$, similar to the laser pulse (see the inset), but on the longer timescale more structures appear for smaller incident energies. With an increase in laser energy, the valley becomes shallower, and the fluctuation on the long timescale diminishes.

\footnotetext{
${ }^{1}$ Department of Physics, Indiana State University, Terre Haute, Indiana 47809, USA, ${ }^{2}$ Department of Physics, Kaiserslautern University of Technology and Research Center OPTIMAS, Box 3049, D-67653 Kaiserslautern, Germany, ${ }^{3}$ Department of Physics and Center for Instruction, Research and Technology, Indiana State University, Terre Haute, Indiana 47809, USA, ${ }^{4}$ Office of the Chancellor and Center for Nanoscience, Departments of Chemistry, Biochemistry and Physics \& Astronomy, University of Missouri-St. Louis, St. Louis, Missouri 63121, USA. *e-mail: gpzhang@indstate.edu.
} 


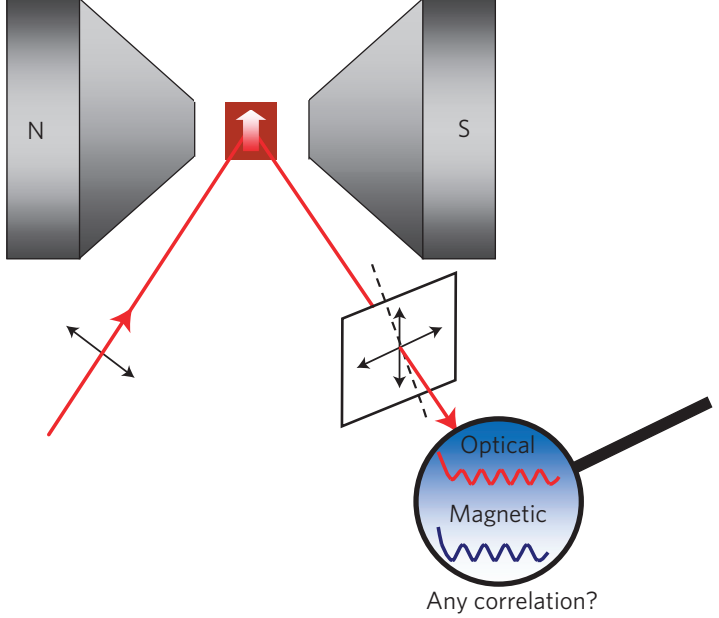

Figure 1 | Schematic geometry of the TRMOKE. Only the optical signal (Kerr ellipticity and rotation) is measured, and how well the signal reflects the magnetization change on the timescale is key to the success of TRMOKE.

The first-order magnetization $\operatorname{Im}\left[M_{x y}^{(1)}\right]$, which is inaccessible experimentally, shows a stronger photon energy dispersion (Fig. 2b): for the photon energy far below $2 \mathrm{eV}, \operatorname{Im}\left[M_{x y}^{(1)}\right]$ also has a similar valley around $0 \mathrm{fs}$; as the energy increases, the valley becomes deeper and wider, and shifts to a later time. However, once it is over $2 \mathrm{eV}$, the valley becomes shallower and shifts to an earlier time, where the original right shoulder now gradually develops into a small hump. As a result, the change in $\operatorname{Im}\left[M_{x y}^{(1)}\right]$ with $\hbar \omega$ leaves a crescent-like trace with respect to time.

A comparison between the magnetic and optical responses leads to our first main result: for laser energy below $2 \mathrm{eV} \operatorname{Im}\left[P_{x y}^{(1)}\right]$ and $\operatorname{Im}\left[M_{x y}^{(1)}\right]$ correlate with each other fairly well, but when the excitation energy is above $2 \mathrm{eV}$ there is a large discrepancy between the magnetic and optical responses. $\operatorname{Im}\left[P_{x y}^{(1)}\right]$ still shows a valley at $0 \mathrm{fs}$, but $\operatorname{Im}\left[M_{x y}^{(1)}\right]$ now shows a peak. This finding is significant, as most laser experiments use only a single central wavelength to probe the dynamics. The threshold energy of $2 \mathrm{eV}$ here reflects the magnetic contribution of the density of states across the Fermi level and may differ in different materials, that is, may be material specific. For a new material, it is necessary to scan different energy windows to avoid an accidental mismatch between polarization and magnetization or bleaching effect. The bleaching effect is defined as how far the magneto-optical response $\left(\operatorname{Im}\left[P_{x y}^{(1)}\right]\right)$ mirrors a true demagnetization $\left(\operatorname{Im}\left[M_{x y}^{(1)}\right]\right)$. This explains a previous experimental finding observed in Fe where the probe wavelength shows a significant effect on the TRMOKE signal ${ }^{28}$, whereas $\mathrm{CoPt}_{3}$ shows a smaller energy dependence ${ }^{7}$. However, is it possible to remove this uncertainty beforehand?

The answer is yes, which was noticed in ferromagnetic perovskite $\mathrm{La}_{0.6} \mathrm{Sr}_{0.4} \mathrm{MnO}_{3}$ (ref. 29), but the authors looked into only the optical response, not the magnetic. We find that the frequency-domain information of both optical and magnetic responses, induced by $\mathrm{cw}$ light, provides good guidance. Figure $2 \mathrm{c}$ shows the off-diagonal optical absorption (solid line) and conductivity (dashed line) as a reference. Figure $2 \mathrm{~d}$ plots the magneto-optical susceptibility, which is defined as (see Supplementary Information)

$$
\chi_{x y, e m}^{(1)}(\Omega)=\sum_{\mathbf{k} ; n m}\left(\rho_{\mathbf{k} ; n n}^{(0)}-\rho_{\mathbf{k} ; m m}^{(0)}\right) \frac{S_{\mathbf{k} ; m n}^{x} D_{\mathbf{k} ; n m}^{y}}{\hbar \Omega-\hbar \omega_{\mathbf{k} ; n m}+i \Gamma_{\mathbf{k} ; n m}}
$$

where $\hbar$ is Planck's constant over $2 \pi$, the summation is over $\mathbf{k}$ points and band states $(n, m), \rho$ is the density matrix, $S^{x}$ is the

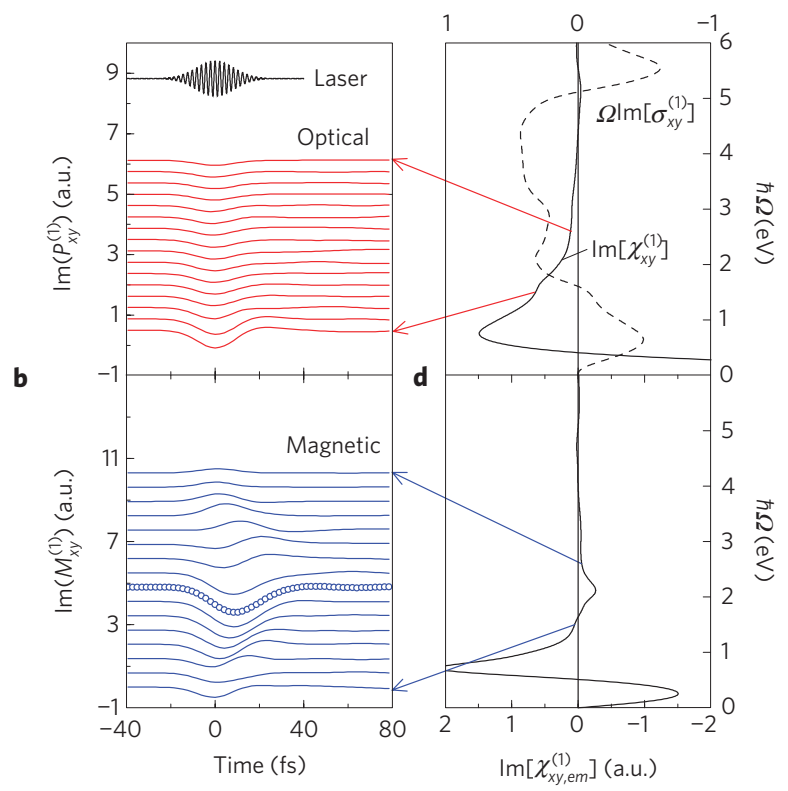

Figure 2 | Laser-induced ultrafast optical and magnetic responses.

a, Time evolution of the first-order off-diagonal polarization as a function of incident photon energy. From bottom to top, the incident photon energies $\hbar \omega$ are $1.5,1.7,1.8,1.9,1.925,1.95,1.975,2.0,2.025,2.075,2.1,2.15,2.3,2.4$ 2.5 and $2.6 \mathrm{eV}$. Inset: laser pulse shape. The laser pulse duration is $12 \mathrm{fs}$, and the laser field strength is $0.05 \mathrm{~V} \AA^{-1}$. b. Off-diagonal magnetization change as a function of time. The open-circled line denotes the result at $\hbar \omega=2.0 \mathrm{eV}$. The change leaves a crescent-like trace. c, Off-diagonal susceptibility (solid line) and conductivity (dashed line) as a function of the probe photon energy $\hbar \Omega$. Note that the $x$ axis is the amplitude of the spectrum, and the $y$ axis is the probe photon energy. $\mathbf{d}$, Magneto-optical susceptibility as a function of probe photon energy $\hbar \Omega$. Four arrows identify where the excitation energy is used in $\mathbf{a}$ and $\mathbf{b}$

$x$-component of the spin matrix, $D^{y}$ is the $y$-component of the dipole operator, $\hbar \omega_{\mathbf{k}: n m}$ is the band energy difference between states $n$ and $m$ and $\Gamma$ is the damping $(0.2 \mathrm{eV})$, which takes into account effects beyond density functional theory. Figure $2 \mathrm{c}$, d explains the energy dependence observed in Fig. 2a,b, respectively. The decrease in the valley with energy in Fig. $2 a$ is directly connected to the decrease in the absorption spectrum in this region, and the energy dependence of $\operatorname{Im}\left[M_{x y}^{(1)}\right]$ is consistent with the change of the magneto-optical susceptibility. The four arrows denote the energy region used to excite the system.

To quantify the correlation between polarization and magnetization, we develop a sensitive phase diagram, a $P_{x y}^{(1)}$-versus- $M_{x y}^{(1)}$ plot, which can monitor phase, amplitude and period differences simultaneously. We align $P$ along the $x$ axis and $M$ along the $y$ axis. Their trace carries rich information about the relation between $P_{x y}^{(1)}$ and $M_{x y}^{(1)}$ (see the inset above Fig. 3c). For instance, if $P$ and $M$ match perfectly, their trace should be a straight line in the first and third quadrants; if they mismatch, the line becomes a loop. The width of the loop reflects the level of the match between $P$ and $M$. If $P$ and $M$ were completely out of phase, the line or loop would fall in the second and fourth quadrants.

Our findings are truly insightful. Figure 3 shows both $P$ and $M$ to start at $-40 \mathrm{fs}$ with zero value and progress in a counter-clockwise fashion, which demonstrates that $P_{x y}^{(1)}$ precedes $M_{x y}^{(1)}$. The arrows represent the time directions, and numbers close to the curve denote the times. The laser pulse duration is $12 \mathrm{fs}$, and the energy is $2 \mathrm{eV}$. The two dashed lines identify the zero values. $P_{x y}^{(1)}$ reaches its negative maximum around $0 \mathrm{fs}$, whereas $M_{x y}^{(1)}$ delays by $10 \mathrm{fs}$, which 

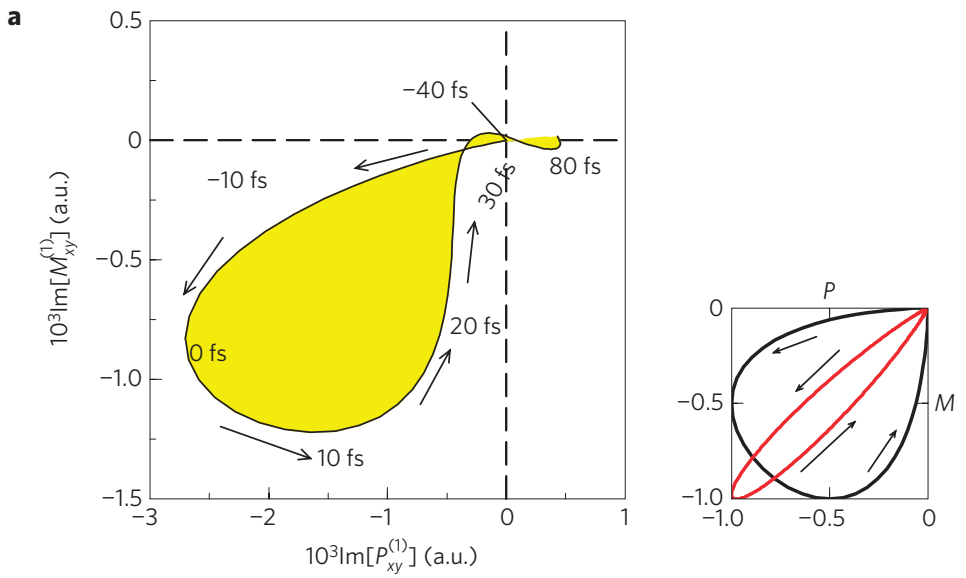

b

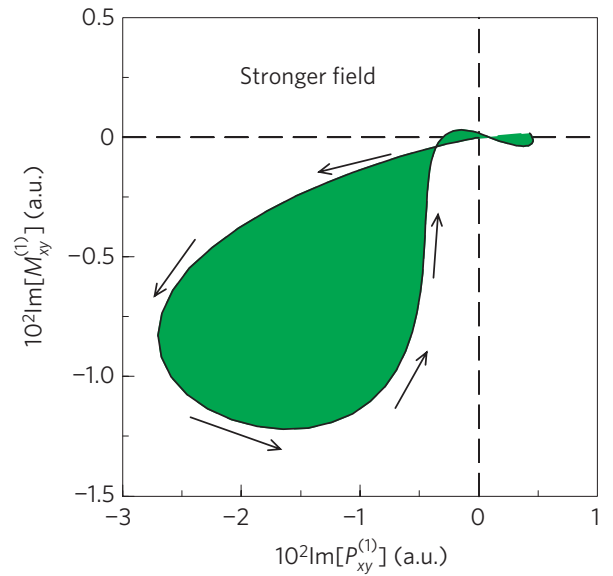

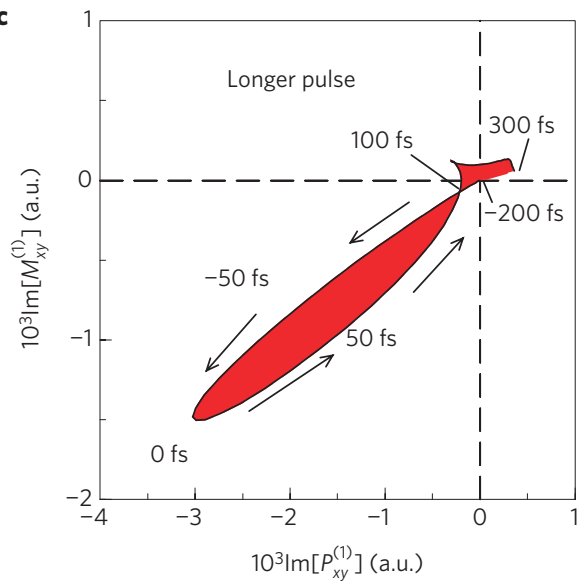

Figure 3 | Monitoring the correlation between polarization and magnetization changes. a-c, Phase diagram of polarization versus magnetization for a laser field amplitude of $0.05 \mathrm{~V} \AA^{-1}$ and duration of $12 \mathrm{fs}$ (a), laser field amplitude of $0.5 \mathrm{~V} \AA^{-1}$ and duration of $12 \mathrm{fs}$ (b) and laser field amplitude of $0.05 \mathrm{~V} \AA^{-1}$ and duration of $64 \mathrm{fs}$ (c). The trace propagates anticlockwise with time. The arrows represent the time direction. The photon energy is $2.0 \mathrm{eV}$. Inset: Phase diagram for two different pulse durations.

is comparable to the charge dephasing time $\mathrm{e}^{30}$. After $30 \mathrm{fs}$, both the magnetic and optical signals come back to zero. This time delay does not lead to a change, even if we increase the laser intensity tenfold from 0.05 to $0.50 \mathrm{~V} \AA^{-1}$ (Fig. 3b) while keeping the pulse duration at $12 \mathrm{fs}$ (see Supplementary Information). Therefore, we arrive at our second conclusion: there is an intrinsic mismatch between the optical and magnetic responses. At the laser energy of $2 \mathrm{eV}$, this mismatch is maximized.

Next, the laser pulse duration has a substantial effect on the correlation between the magnetic and optical responses. When we increase the laser duration to $64 \mathrm{fs}$, we find that the original loop collapses into a narrow and symmetric loop (see Fig. 3c), where both $P_{x y}^{(1)}$ and $M_{x y}^{(1)}$ reach their respective negative maxima at the same time and follow each other faithfully over $300 \mathrm{fs}$. This reveals a new paradigm for the magneto-optical Kerr effect: the optical response reflects the magnetic signal if the laser pulse is longer than the charge dephasing time $e^{30}$. As most of the experiments use much longer pulses than ours, the observed Kerr signal does reflect the magnetization change, provided that the time dependence is dominated by the off-diagonal polarization.

The reason why the pulse duration has a substantial influence on the correlation between the magnetic and optical responses can be understood from the nature of state excitation and dephasing. It is known that states with larger transition energies dominate the dynamics for the first few femtoseconds. However, states which make substantial magnetic contributions have lower transition energies. A long laser pulse can wait for those states of large transition energies to decay before it induces a substantial magnetic response. Once the initial dephasing is over, the optical and magnetic responses are driven by similar sets of states and tend to correlate with each other much better. This result finally resolves a decade-long debate as to whether TRMOKE really probes the magnetization, and also, importantly, it establishes a solid theoretical foundation for femtomagnetism.

Received 22 October 2008; accepted 20 May 2009; published online 14 June 2009

\section{References}

1. Argyres, P. N. Theory of the Faraday and Kerr effects in ferromagnetics. Phys. Rev. 97, 334-345 (1955).

2. Erskine, J. L. \& Stern, E. A. Magneto-optic Kerr effects in gadolinium. Phys. Rev. B 8, 1239-1255 (1973).

3. Beaurepaire, E., Merle, J.-C., Daunois, A. \& Bigot, J.-Y. Ultrafast spin dynamics in ferromagnetic nickel. Phys. Rev. Lett. 76, 4250-4253 (1996).

4. Zhang, G. P., Hübner, W., Beaurepaire, E. \& Bigot, J.-Y. Laser-induced ultrafast demagnetization: Femtomagnetism, a new frontier? Topics Appl. Phys. 83, 245-289 (2002).

5. Koopmans, B., van Kampen, M., Kohlhepp, J. T. \& de Jonge, W. J. M. Ultrafast magneto-optics in nickel: Magnetism or optics? Phys. Rev. Lett. 85, 844-847 (2000).

6. Guidoni, L., Beaurepaire, E. \& Bigot, J.-Y. Magneto-optics in the ultrafast regime: Thermalization of spin populations in ferromagnetic films. Phys. Rev. Lett. 89, 017401 (2002).

7. Bigot, J.-Y., Guidoni, L., Beaurepaire, E. \& Saeta, P. N. Femtosecond spectrotemporal magneto-optics. Phys. Rev. Lett. 93, 077401 (2004). 
8. Wilks, R. et al. Investigation of ultrafast demagnetization and cubic optical nonlinearity of $\mathrm{Ni}$ in the polar geometry. J. Appl. Phys. 95, 7441-7443 (2004).

9. Comin, A., Rossi, M., Mozzati, C., Parmigiani, F. \& Banfi, G. P. Femtosecond dynamics of Co thin films on Si support. Solid State Commun. 129, 227-231 (2004).

10. Stamm, C. et al. Femtosecond modification of electron localization and transfer of angular momentum in nickel. Nature Mater. 6, 740-743 (2007).

11. Stanciu, C. D. et al. All-optical magnetic recording with circularly polarized light. Phys. Rev. Lett. 99, 047601 (2007).

12. Zhang, G. P. \& Hübner, W. Laser-induced ultrafast demagnetization in ferromagnetic metals. Phys. Rev. Lett. 85, 3025-3028 (2000).

13. Lefkidis, G. \& Hübner, W. First-principles study of ultrafast magneto-optical switching in NiO. Phys. Rev. B 76, 014418 (2007).

14. Gomez-Abal, R., Ney, O., Satitkovitchai, K. \& Hübner, W. All-optical subpicosecond magnetic switching in $\mathrm{NiO}(001)$. Phys. Rev. Lett. 92, 227402 (2004)

15. Hohlfeld, J., Matthias, E., Knorren, R. \& Bennemann, K. H. Nonequilibrium magnetization dynamics of nickel. Phys. Rev. Lett. 78, 4861-4864 (1997); erratum 79, 960-960 (1997).

16. Regensburger, H., Vollmer, R. \& Kirschner, J. Time-resolved magnetization-induced second-harmonic generation from the $\mathrm{Ni}(110)$ surface. Phys. Rev. B 61, 14716-14722 (2000).

17. Malinowski, G. et al. Control of speed and efficiency of ultrafast demagnetization by direct transfer of spin angular momentum. Nature Phys. 4 855-858 (2008).

18. Radu, I. et al. Laser-induced magnetization dynamics of lanthanide-doped permalloy thin films. Phys. Rev. Lett. 102, 117201 (2009).

19. Kurkin, M. I., Bakulina, N. B. \& Pisarev, R. V. Transient inverse Faraday effect and ultrafast optical switching of magnetization. Phys. Rev. B 78 134430 (2008).

20. Hübner, W. \& Zhang, G. P. Ultrafast spin dynamics in nickel. Phys. Rev. B 58, R5920-R5923 (1998).

21. Oppeneer, P. M. \& Liebsch, A. Ultrafast demagnetization in Ni: Theory of magneto-optics for non-equilibrium electron distributions. J. Phys. Condens. Matter 16, 5519-5530 (2004).

22. Vernes, A. \& Weinberger, P. Formally linear response theory of pump-probe experiments. Phys. Rev. B 71, 165108 (2005).

23. Zhang, G. P. Laser-induced orbital and spin excitations in ferromagnets: Insights from a two-level system. Phys. Rev. Lett. 101, 187203 (2008).

24. Zhang, G. P. \& George, T. F. Total angular momentum conservation in laser-induced femtosecond magnetism. Phys. Rev. B 78, 052407 (2008).
25. Zhang, G. P., Bai, Y., Hübner, W., Lefkidis, G. \& George, T. F. Understanding laser-induced ultrafast magnetization in ferromagnets: First-principles investigation. J. Appl. Phys. 103, 07B113 (2008).

26. Bai, Y. \& Ward, R. C. Parallel block-tridiagonalization of real symmetric matrices. J. Parallel. Distrib. Comput. 68, 703-715 (2008).

27. Blaha, P., Schwarz, K., Madsen, G. K. H., Kvasnicka, D. \& Luitz, J. WIEN2k, An Augmented Plane Wave + Local Orbitals Program for Calculating Crystal Properties (Karlheinz Schwarz, Techn. Universität Wien, 2001).

28. Kampfrath, T. et al. Ultrafast magneto-optical response of iron thin films. Phys. Rev. B 65, 104429 (2002).

29. Ogasawara, T. et al. Photoinduced spin dynamics in $\mathrm{La}_{0.6} \mathrm{Sr}_{0.4} \mathrm{MnO}_{3}$ observed by time-resolved magneto-optical Kerr spectroscopy. Phys. Rev. B 68, 180407(R) (2003).

30. Bigot, J.-Y. Femtosecond magneto-optical processes in metals. C. R. Acad. Sci. Paris, t. Sèrie IV 2, 1483-1504 (2001).

\section{Acknowledgements}

This work was supported by the US Department of Energy under contract No DE-FG02-06ER46304 and US Army Research Office under contract

W911NF-04-1-0383, and was also supported by a Promising Scholars grant from Indiana State University. In addition, we acknowledge part of the work as done on Indiana State University's high performance computers. This research used resources of the National Energy Research Scientific Computing Center, which is supported by the Office of Science of the US Department of Energy under contract No DE-AC02-05CH11231. W.H. and G.L. acknowledge support from Priority Programmes 1133 and 1153 of the German Research Foundation. Initial studies used resources of the Argonne Leadership Computing Facility at Argonne National Laboratory, which is supported by the Office of Science of the US Department of Energy under contract No DE-AC02-06CH11357.

\section{Author contributions}

G.P.Z. drafted the paper, and W.H., G.L., Y.B. and T.F.G. modified it. G.P.Z. computed the results, and G.P.Z., W.H. and G.L. analysed the data. Y.B. implemented the parallelization of the source code.

\section{Additional information}

Supplementary information accompanies this paper on www.nature.com/naturephysics. Reprints and permissions information is available online at http://npg.nature.com/ reprintsandpermissions. Correspondence and requests for materials should be addressed to G.P.Z. 